\title{
An evaluation of combined narrative exposure therapy and physiotherapy for comorbid PTSD and chronic pain in torture survivors
}

Iselin Dibaj, cand.psychol.*, Joar Øveraas Halvorsen, cand.psychol., PhD**, Leif Edward Ottesen Kennair, cand.psychol., PhD***, Håkon Inge Stenmark, cand.psychol. PhD****

\section{Key points of interest}

- Novel treatment approach coupling narrative exposure therapy with physiotherapy.

- Small detailed study with six torture survivors.

- Torture survivors suffering from high symptom loads can achieve good outcomes when working in parallel with traumas and pain.

- Individual differences between the six different cases; no treatment works for all patients.

\section{Abstract}

Introduction: Torture is associated with adverse health consequences, with especially high rates of PTSD, depression and chronic pain. Despite increased awareness of the relationship between pain and posttraumatic

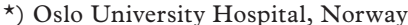

$\star \star)$ Nidaros District Psychiatric Centre, St. Olavs Hospital, Trondheim University Hospital

$\star \star \star)$ Department of Psychology, Norwegian University of Science and Technology (NTNU)

$\star \star \star \star)$ Center on violence, traumatic stress and suicide prevention in Mid-Norway, St. Olavs Hospital,

Trondheim University Hospital
}

Correspondence to: iselin.dibaj@gmail.com symptoms, and the accompanying need for effective treatment strategies, few studies have examined an integrated treatment of comorbid PTSD and pain. Methods: In this study, using an A-B case series design with three and six month follow-up, six refugee torture survivors with comorbid PTSD, depression and chronic pain received 20 sessions of Narrative Exposure Therapy (NET) and 10 sessions of physiotherapy. Outcome variables included symptoms of PTSD and depression, pain intensity, physical functioning and quality of life. Symptoms of PTSD and pain were also rated after each treatment session. Results: Two patients achieved clinically significant reduction in symptoms of PTSD. Only one patient achieved clinically significant change in depressive symptoms, and two experienced clinically significant reduction in pain intensity. Clinical descriptions of the course of treatment for all patients are provided. Discussion and Conclusions: Despite its limitations, the study suggests that some torture survivors who suffer high symptom loads may benefit from a combined treatment of NET and physiotherapy. Appreciating individual differences and how they affect treatment can provide valuable insight and inform clinicians working with torture survivors. Directions for future research 
regarding the improvement of rehabilitation strategies of torture survivors are discussed, and highlighted through descriptions from the six therapy cases.

Keywords: PTSD, chronic pain, torture survivors, narrative exposure therapy, physiotherapy, treatment outcome

\section{Introduction}

Torture is associated with adverse mental health consequences, such as high rates of symptoms of PTSD, depression and chronic pain, ${ }^{1,2}$ and low levels of functioning and quality of life. ${ }^{3}$ A number of studies have investigated the link between pain and PTSD, including amongst torture survivors specifically. ${ }^{4}$ Chronic pain is highly prevalent in torture survivors, ${ }^{4}$ often co-occurs with PTSD and is associated with poorer prognosis. ${ }^{1}$ Trauma survivors who have suffered trauma-related bodily injury have an eight-fold higher risk for developing PTSD, ${ }^{5}$ which could potentially explain the higher incidence of chronic pain and PTSD amongst torture survivors. Defrin et al. ${ }^{6}$ conclude that "torture survivors exhibit generalized diminished pain modulation and increased excitability. These alterations in the function of the pain system may underlie the chronic pain decades after the torture" (p549). Furthermore, pain-related fear has been found to be a predictor of chronicity of pain symptoms, ${ }^{7}$ and PTSD is a higher risk factor for developing pain than the experience of trauma by itself. ${ }^{8}$ PTSD has gained support as a risk factor contributing to more severe pain experience, poorer treatment outcome and greater pain-related disability. ${ }^{9}$ A number of theoretical models explain the relationship between chronic pain, PTSD and depression. ${ }^{4,10,11}$ The theoretical models and empirical studies give rise to several potential treatment implications. First, the effective treatment of PTSD could lead to a reduction of symptoms of chronic pain and depression and thus improve pain rehabilitation outcome in patients with chronic comorbid affective and pain symptoms (for a review, see reference 12). Second, PTSD and chronic pain can be treated simultaneously by targeting shared vulnerabilities ${ }^{11}$ or through integrated treatments. ${ }^{41}$ Third, successful treatment of PTSD symptoms may lead to increased quality of life and functioning.

Trauma-focused cognitive behavioral therapy (TF-CBT) is currently the best documented treatment for PTSD. ${ }^{13}$ Recently, a Cochrane review on effective interventions for torture survivors concluded that narrative exposure therapy (NET) and TF-CBT had moderate effects on posttraumatic symptoms and distress six months after treatment, but the authors stressed the substantial limitations of the current evidence base. ${ }^{14}$ Another recent review classified CBT and NET as promising interventions for torture survivors suffering from PTSD, depression or pain. ${ }^{15}$

According to guidelines for physiotherapy for torture survivors, physiotherapy and psychotherapy should be implemented in parallel. ${ }^{16}$ To our knowledge, few studies exist which investigate the effects of physiotherapy, or combined physiotherapy and psychotherapy, for torture survivors. However, Carlsson et al. ${ }^{17}$ reviewed the effect of multimodal treatment implemented for $71 \%$ of a sample of patients at a specialized center for trauma and torture. The treatment included psychotherapy, physiotherapy, medical attention and social assistance with a mean of 35 sessions in total. No treatment effect was found for PTSD, depression or quality of life. At the 23-month follow-up, Carlsson et al. ${ }^{18}$ found that approximately $1 / 3$ of the participants reported reliable 
symptom improvement as measured by self-report.

\section{Aims of this study}

For the present study, physiotherapy was combined with NET in an effort to alleviate emotional distress and pain, as well as to increase quality of life and functioning, in survivors of torture presenting with comorbid pain and PTSD. Specific study aims were:

1. To run a preliminary evaluation of the potential efficacy of a combination treatment for torture survivors, targeting both posttraumatic symptoms and pain.

2. To highlight individual variation in response to the treatment, and discuss potential predictors, based on the reported clinically significant change and an analysis of the six case illustrations.

3. To explore and present topics for future research that may emerge from the descriptions of six therapy cases.

\section{Method}

\section{Design}

To examine the treatment effect of a combination of NET and physiotherapy for torture survivors with PTSD and pain, an $\mathrm{A}-\mathrm{B}-\mathrm{design}{ }^{19}$ with a three and six month follow-up was chosen. A case series design is deemed suitable for preliminary examinations of novel treatment approaches. The patients were divided into three different groups, where the onset time of physiotherapy varied between after the third, sixth and ninth NET session in order to examine whether there was an obvious effect of physiotherapy. Three experienced professionals were involved in the psychological

\footnotetext{
${ }^{\mathrm{i}}$ http://rvtsmidt.no/english/
}

assessment and NET treatment, but the assessor and therapist were independent of each other. All patients had the same physiotherapist, but were assessed by an independent physiotherapist.

\section{Selection of Participants}

The recruited patients were referred to the outpatient clinic for refugees connected to The Regional Center on Violence, Traumatic Stress and Suicide Prevention, Mid-Norway. ${ }^{\mathrm{i}}$ Collaboration between the referring clinician, hospital, general practitioner and Trondheim Municipality Refugee Health Team assured that the patients had been assessed according to the Istanbul Protocol. ${ }^{20}$ Patients who met the inclusion criteria were invited to participate, namely, that they were 18 years or older, had experienced torture, reported pain symptoms and fulfilled the diagnostic criteria for PTSD according to DSM-IV. Exclusion criteria were psychosis, high suicide risk or a serious drug/alcohol addiction. Information about the project, including the possibility of withdrawing at any time, was presented in writing in the language of each patient, as well as being orally translated. All patients signed an informed consent. Subsequently, patients were further assessed for PTSD, depression, psychiatric comorbidity, pain symptoms, functioning and health-related quality of life. The study was approved by the Regional Committee for Medical and Health Research Ethics.

Patients

Initially, eight patients were enrolled in the project. As two withdrew before completion, only six patients are included in the data analysis. The clinical descriptions below illustrate different clinical presentations, challenges and outcomes in treatment of torture survivors suffering from PTSD and pain. All six patients had refugee status when 
treatment started. The number of traumatic experiences apart from the torture was high, with a mean of $7.83(\mathrm{SD}=2.86)$. The following case descriptions are based on data from the pre-test assessment. All patients reported substantial sleep disturbances, and all except for patient 5 , had economic problems.

\section{Attrition and Drop-out}

Two patients were excluded from the project: One, because of psychotic symptoms, which at first did not seem substantial; and, another, because of poor attendance despite a proclaimed intention to continue treatment. Both patients were offered a different type of treatment.

\section{Procedure}

After the first assessment and prior to treatment onset, two baseline assessments were performed; after one and three weeks. After the baseline period, the patients engaged in approximately 20 sessions of NET (90 minutes each) and 10 sessions of physiotherapy (60 minutes each). Symptoms of PTSD and pain were assessed at the end of each session by self-report. In addition, the therapists filled out a NET process form after each session, to ensure adherence and document compliance to the NET treatment manual. There were two follow-up assessments, three and six months after treatment. Information about changes in refugee status, marital status, living condition and social activity was also collected. One item from the Brief Pain Inventory (BPI), the Numeric Rating Scale (NRS-11) and the Posttraumatic Diagnostic Scale (PDS) were administered after each treatment session, whereas all other instruments were administered prior to treatment and at the two follow-up assessments. All patients except for patient 3 had an interpreter present during the therapy sessions. Each patient had the same interpreter in all their treatment sessions.

\section{Narrative Exposure Therapy (NET): NET is a} trauma-focused therapy that incorporates principles from prolonged exposure and testimonial therapy. ${ }^{21}$ It was developed specifically for refugees that have experienced multiple traumatic events, such as persecution, flight and torture. NET is based on dual processing theory and aims to contextualize fragmented, traumatic memories. Usually, NET consists of eight to ten sessions, while in this study we have doubled the number of sessions due to the complexity of severe trauma symptoms and chronic pain. The first session is devoted to make a lifeline where the patient identifies and labels traumatic experiences over the course of their lives. In subsequent sessions, the patient and therapist collaboratively record and read the patient's life narrative and prolonged exposure techniques are applied for traumatic memories.

Physiotherapy: All patients received treatment by the same physiotherapist, who had several years of experience in working with refugees and torture survivors. The treatment was individually tailored according to the different needs of each patient.

\section{Instruments ${ }^{\mathrm{ii}}$}

Before treatment and at the follow-up assessments, we administered the ClinicalAdministered PTSD Scale (CAPS) to diagnose and assess severity levels of PTSD, and the Hamilton Rating Scale for Depression (HRSD) to evaluate the severity level of depression. The Norwegian Pain Association's Minimum Inventory for Pain Patients

\footnotetext{
ii Detailed descriptions of the instruments used can be provided upon request.
} 
Table 1: Means and Standard Deviations

\begin{tabular}{lcccccc}
\hline & \multicolumn{2}{c}{ Pre-test } & \multicolumn{2}{c}{ 3 months FU } & \multicolumn{2}{c}{ 6 months FU } \\
\hline MAPS & M & SD & M & SD & M & SD \\
HRSD & 80.83 & 18.63 & 60.33 & 25.56 & 54.83 & 31.24 \\
BPI ${ }^{a}$ & 18.67 & 4.93 & 14.5 & 5.58 & 13.0 & 5.66 \\
\hline
\end{tabular}

$a=n=4, C A P S=$ Clinician-Administered PTSD Scale for DSM-IV, HRSD = Hamilton. Rating Scale for Depression, BPI = Brief Pain Inventory

(NOSF-MISS) was used to assess factors such as pain intensity (BPI), physical functioning, health-related quality of life, sleep difficulties and financial struggles. In the present study, the MINI International Neuropsychiatric Interview (MINI) was conducted to diagnose depression, to assess eventual comorbid psychiatric diagnoses, and to ensure that the patients did not meet the exclusion criteria. Socio-demographic questions were asked about variables such as age, sex, nationality, refugee status, education level, living conditions, social network and period of time in Norway.

NET Adherence and Compliance: Adherence and compliance with the NET manual were rated through a self-report questionnaire, which the therapists completed after each session. They reported whether the therapeutic tasks in the manual were completed or not, how the patient responded to exposure, and whether time was used on other activities or to discuss current stressors.

\section{Data Analysis}

Visual analysis was chosen as it is recommended as the primary method for this type of study design, rather than the performance of statistical analyses. ${ }^{22}$ It allows for an informal judgment of whether a clear treatment effect can be seen, and will, according to Parsonson and Baer, ${ }^{23}$ reveal any treatment effect solid enough to be of importance for clinicians. In addition, the individual results and the extent of any clinically significant change for all six patients is reported. The raw scores are available on request, making it possible to compute effect sizes as recommended by Brossart et al. ${ }^{24}$

\section{Results}

The symptom progression of pain intensity and PTSD symptoms for the individual patients is presented in Figure 1. Means and standard deviations for symptoms of PTSD, depression and pain intensity are presented in Table 1.

\section{Clinical descriptions}

Patient 1: Saleem: Saleem iii was in his 30s, was from the Middle East, and had been in Norway for 10 years. He lived with his wife and two children, and attended school. At seventeen, he was arrested and tortured for the first of several times, after he had been politically active for many years. In total, he spent 7.5 months in captivity and was tortured during 5.5 of them. The torture

\footnotetext{
iii All patient names are pseudonyms. Pseudonyms were selected from amidst name registers of the patient's respective country of origin. Cross-checking was then performed to ensure that the selected pseudonym also was in use in neighboring countries, to preclude recognition based on nationality.
} 
Figure 1: Symptom progression of pain intensity and PTSD symptoms by patient and session
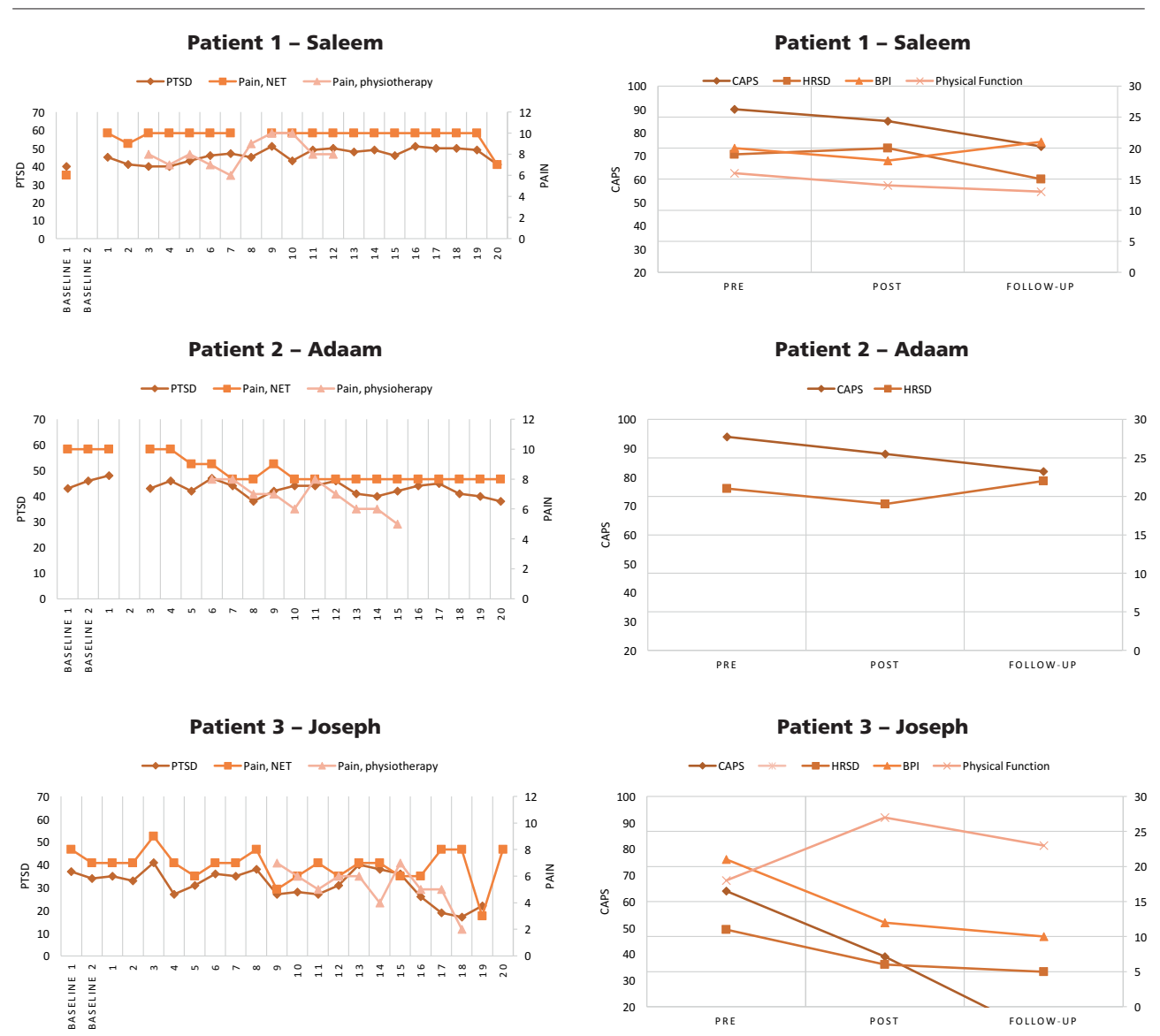

'PTSD'= PDS Scores, 'Pain, NET'= BPI scores, 'Pain physiotherapy'= NSR-11 scores, PDS = PTSD Diagnostic Scale. NSR-11 = Numeric Rating Scale.

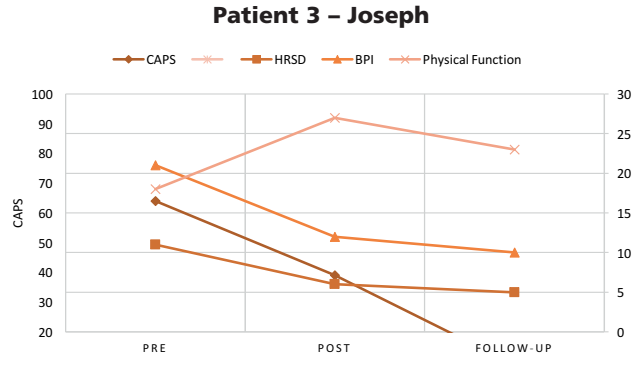

'CAPS' = Clinician-Administered PTSD Scale for DSM-IV, 'HRSD' = Hamilton Rating Scale for Depression, 'BPI' = Brief Pain Inventory, Physical Function is a subscale in the NOSF-MISS.

consisted of blindfolding, beatings until he lost consciousness, threats towards self and family members, whipping, isolation, deprivation, water torture, suspension from different limbs, and degrading insults. He fulfilled the criteria for PTSD, a moderate depressive episode, and had chronic pain that started after the torture, located in his head, face, jaw, knees, feet, ankles, elbows, lower back and legs. He suffered damage that

required surgery that led to painful repercussions. Also, he was overweight, which added additional strain on his feet, knees and ankles.

Overall, few treatment sessions fully complied with the NET manual, and the lifeline was not completed until the sixth session. Saleem talked in a detailed manner, and the therapist often found it challenging to moderate his flow of words as well as engaging him in exposure. Filling out 
Figure 1: Symptom progression of pain intensity and PTSD symptoms by patient and session
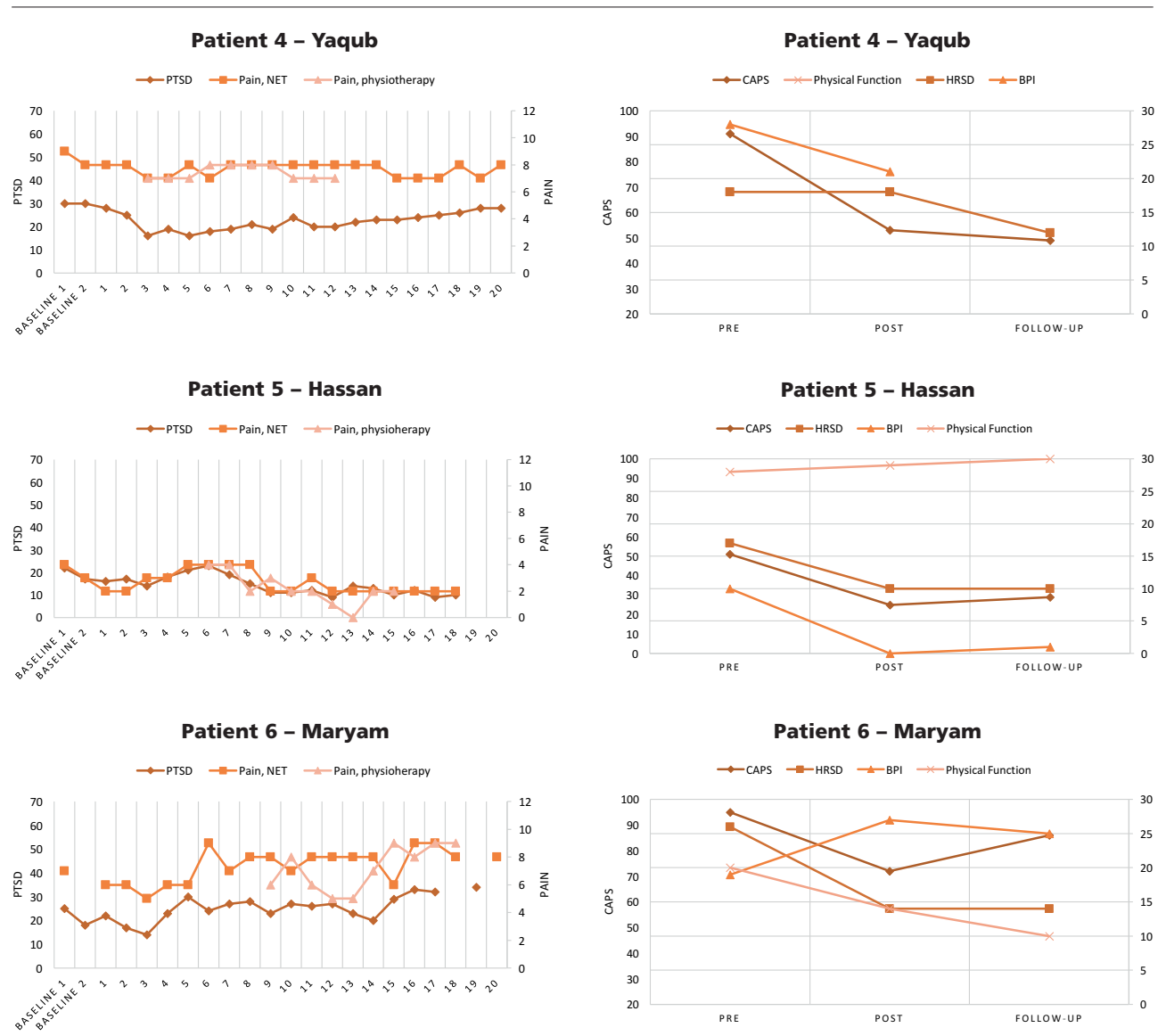

'PTSD'= PDS Scores, 'Pain, NET'= BPI scores, 'Pain physiotherapy'= NSR-11 scores, PDS $=$ PTSD Diagnostic Scale. NSR-11 = Numeric Rating Scale.

'CAPS' = Clinician-Administered PTSD Scale for DSM-IV, 'HRSD' = Hamilton Rating Scale for Depression, 'BPI' = Brief Pain Inventory, Physical Function is a subscale in the NOSF-MISS.

baseline recordings sometimes took half of the session, and exposure often closed prematurely, as they ran out of time. Saleem had a number of current stressors he wanted to address in therapy, including the family's living situation, his pain symptoms and physical disabilities. His physical state also made it difficult for him to sit still in the same position for longer periods. He still met criteria for PTSD at the final follow-up.

When starting physiotherapy, Saleem was physically inactive and had some physical disabilities, which impeded him in benefitting from pain-reducing treatments besides hydrotherapy and sitting exercises. He was cooperative and motivated for the treatment. His initial scepticism towards hydrotherapy, as he could not swim, ceased after a few sessions, and he enjoyed reduced pain intensity after five sessions. Still, his 
level of functioning did not improve much. Mid-treatment, he had surgery on a foot, which led to a relapse of pain intensity, as the pain increased and became worse than before starting treatment. However, the pain returned to baseline towards the last session. His final remarks were "I feel sorry for my party fellows, as they do not receive the kind of help that I do now".

Patient 2: Adaam: Adaam was in his 60s from the Caucasus, and had been in Norway for eight years, living with his wife. For many years, he was politically active through critical writings about the government, which led to him being arrested and tortured twice, both episodes lasting a day. The torture consisted of bindings, beatings and water torture - in addition, they tortured a close family member. After the last episode, he fled with his family, living in hiding and constant fear for three years until they came to Norway. Adaam described himself as functioning well after the torture, but he had had reduced functioning for the previous seven years. His pain symptoms began after the torture, were growing increasingly worse and located in his head, chest, stomach, thighs, neck, lower back, shoulder and leg. He also suffered from severe toothache that required painful surgery. Adaam fulfilled the criteria for PTSD, a moderate depressive episode, social phobia and lifetime panic disorder with agoraphobia.

Overall, most sessions complied with the NET manual, and the therapeutic relationship was described as good. Adaam was a good storyteller with a detail-rich manner of speaking; this sometimes impeded activation of affect as required in exposure therapy. In fact, he often lectured about his torture experiences, even though this made him anxious. How this influenced him was reflected upon throughout the therapy. Although, when the therapist repeatedly pointed out how this style may lead to avoidance and repeated the treatment rationale, Adaam cooperated. In the final stages of treatment,
Adaam expressed that he wanted a full disability pension because of his severe pain. He concluded, "This treatment has helped me see how the pieces fit together, but now I don't think there is anything left to do". He still met the criteria for PTSD at the final follow-up.

Adaam was highly motivated to receive the physiotherapy. He emphasized that he saw himself as a survivor of torture, not a victim. $\mathrm{He}$ complied with a physical exercise program that he performed both in and between sessions. In addition, he engaged in hydrotherapy. Adaam's level of functioning improved, and his pain was reduced from an extreme to a moderate level of pain. His concluding remarks were "I am proud of surviving, and will manage on my own now, because you taught me what to do when in pain”.

Patient 3: Joseph: foseph was in his 40s from Central Africa, and came to Norway 10 years ago. He lived with his wife and had regular contact with his child. Foseph had two degrees, a paid job and volunteered in an organization, but was on a sick leave. At a young age, he became politically active with the resistance party, for which he was arrested several times. When in prison, the torture went on for two months. The torture included stabbing, isolation, deprivation, threats and positional torture. He also experienced violence from adults during childhood, a traffic accident and witnessing the torture and murder of others. Foseph described posttraumatic symptoms beginning when he was released from captivity 15 years ago, increasing when he experienced a difficult time living in poverty in exile, with no social network. He fulfilled the criteria for PTSD, a mild depressive episode and had pain in his head, chest, hands, shoulders, elbows, upper and lower back - the latter appeared diffuse.

In the beginning, foseph exhibited avoidance during exposure, manifested in sudden changes of topic to daily life stressors. In session 8, he 
reported increased symptom load or retraumatization after exposure to childhood memories and encounters with other refugees' stories through his volunteer work. Two sessions focused on these reactions, before returning to exposure. During exposure, he sometimes felt bodily pain and headaches, and the NET therapist and physiotherapist cooperated on this matter between sessions. A few sessions included cooperation with the physiotherapist, and some focused on reflections on meaning, identity, and religion. foseph stated that this was the first time anyone had talked with him about his traumatic experiences and that it was a relief to have done it. He did not meet criteria for PTSD at the final follow-up.

foseph was highly committed to the physiotherapy, and was motivated to restore his ability to help others by fighting injustice and poverty. He completed an exercise program in and between sessions, focused on reducing and coping with his pain. The physiotherapist described foseph as having exercised thoroughly, with reduced pain and improved functioning. At the end of treatment, Foseph announced "I found myself in the project, as well as the way towards becoming independent, and I now know how to ease my own pain".

Patient 4: Yaqub: Yaqub was in his 30s from the Caucasus, and had been in Norway for 10 years. He lived with his wife and children, had a university degree and a paid job, but was on sick leave. He described a secure childhood until he turned 15, when war hit his village. Due to political activity in his family, he was arrested and tortured twice, both episodes lasting for one day. The torture included electricity, beatings to the head and body, degrading comments, a tight rubber band around his head, water torture, physical abuse with canes, weapon threats, being stripped naked, having rocks thrown at him, cigarette stumping, falanga, suspension from the arms and buried alive in a dark hole.Yaqub described posttraumatic and pain symptoms shortly after the torture, but loss in function for only the previous three years. He fulfilled the criteria for PTSD, a moderate depressive episode and had pain in his head, hands, knees, feet, neck, shoulders, elbows, lower back, and legs.

In Yaqub's therapy sessions, the exposure complied with the NET manual for most sessions, and the therapeutic relationship was described as good. However, exposure was often closed prematurely or characterized by lack of activated affect in session. The therapist reported time as a hindering factor, since they seldom had time for in-depth exposure after reading through the narrative at the beginning of the sessions. Yaqub also arrived late a few times. During exposure for the torture sequences, Yaqub experienced physical pain but rarely displayed emotional distress. Some sessions concentrated exclusively on positive events (flowers), and a few were focused on current stressors, including the family's living situation, Yaqub's sleep problems, and everyday triggers of posttraumatic symptoms. At the end of the treatment, he communicated that he did not feel that he had improved, however, he did not meet criteria for PTSD at the final follow-up.

When starting the treatment, Yaqub was not motivated for physiotherapy and expressed lack of hope for improvement. Instead of physical therapy, he wanted to focus on talking about the torture, and understanding why the torturers had localized specific body parts targeted with specific methods. Yaqub was anxious about hydrotherapy because of the water torture he had endured, and he did not participate in the first two sessions. In the third session, he complied and enjoyed the exercises in the warm water.

Patient 5: Hassan: Hassan was in his 30s, from the Middle East and had been in Norway for a year. He lived with his wife and child, and was taking Norwegian classes. When Hassan was a teenager, he started military training and 
became a guerilla soldier for three years. Subsequently, he was imprisoned five times in two different countries. During one of the periods of detention, he was tortured for 12 days, in another for 20. The torture consisted of psychological pressure, breaking of his arm, being stripped naked, electric shocks, deprivation, and living in unsanitary conditions. He described psychological struggles prior to the torture, but that the posttraumatic symptoms began afterwards. Hassan was diagnosed with PTSD, a moderate depressive episode, and had pain and reduced functioning in his, neck, shoulders, chest, lower and upper back - all worsening in wintertime. He also had pain in his head and legs, and suffered additional pain related to medical treatment of somatic disease.

Early in treatment, Hassan expressed ambivalence about receiving treatment, but he decided to give it a try. Simultanously, he was in the middle of a divorce, and 10-20 minutes of most therapy sessions focused on this process or statements and applications related to Hassan's living situation, school and work practice. During exposure, he sometimes experienced headaches and bodily pain, which subsequently the therapist discussed with the physiotherapist between sessions. About half of the sessions complied with the NET manual, but paused halfway as Hassan confided that he had a marijuana abuse problem. Associations between drug use, PTSD and its treatment were addressed, referral to an addiction treatment center was discussed. Hassan decided to try to quit on his own. Subsequently, he complied in all sessions, but found it challenging to endure exposure in the end, as he felt most of his symptoms were gone and instead wanted to focus on his future. Some sessions were focused on how the trauma had influenced his family life, religion, identity, existential issues, and how he experienced meaning after receiving therapy. At the end of treatment, Hassan had successfully ended his marijuana abuse and was working on the relationship with his wife, who was invited for the final therapy session. He did not meet the criteria for PTSD at the final follow-up.

In the physiotherapy, despite his reduced physical function, Hassan was proud of his refusal to let this hinder him from being physically active and performing activities of daily living. He was cooperative and motivated for the treatment and had physical therapy in all sessions. After treatment, his pain intensity level was halved. He expressed that he wanted to return to his home country to show that he is healthy, and described his pain as "small troubles that can't wear down a former guerilla soldier".

Patient 6: Maryam: Maryam was in her 30s from the Middle East, had been in Norway for a year, was divorced and lived with her child. She had completed high school and was studying. Maryam and her family were politically active, which led to her being arrested with her daughter while in her 20s. They were held captive for 1.5 months and moved between different prisons. In prison, they both fell ill, but were refused food and medical treatment. The torture consisted of beatings with a baton, falanga, deprivation, isolation, refused clothing, being bound, and witnessing the torture of others. She reported struggling with anxiety and depression before, while her posttraumatic symptoms began after the torture. She fulfilled the criteria for PTSD, panic disorder, recurrent depressive disorder (current episode moderate), social phobia and pain in her head, chest, abdomen, hands, knee, foot, neck, hip, shoulder, upper and lower back. She also suffered from fatigue, headaches and rheumatic symptoms, fibromyalgia, a somatic disease and arthritis. Maryam had no social network in the region besides her daughter.

In the early stages of therapy, Maryam complied with the NET activities and expressed motivation for exposure. During exposure, she often exhibited avoidance, manifesting in excessive details, and she did not always reach 
optimal activation of affect. From the fifth session, more time focused on current stressors; Maryam had received news that her mother had fallen seriously ill. This was especially difficult for Maryam, as she was unable to return to visit her and had no social network to turn to. Before the $16^{\text {th }}$ session, she was in a work-related accident, and as a consequence suffered back damage, further decreased physical function and increased pain and distress. She also hit her head in the fall. In sessions, Maryam struggled to conceal her pain, reported constant fatigue, was frequently cold and found walking very painful. Therefore, the therapist used some time to work on sitting posture, relaxation, grounding, and provided blankets. Mid-therapy, Maryam wanted to move to a different part of the country, where she had family. The therapist helped her to arrange this, and considered the availability of social support to be of great importance for Maryam and her psychological health. Maryam expressed that it felt good to have talked about the traumas. She still met criteria for PTSD at the final follow-up.

In the physiotherapy, Maryam was distressed because of high pain intensity in her whole body, both musculoskeletal and rheumatic in nature. Despite this, as well as constant fatigue and headaches, she complied with and was motivated for the physical exercise program in the beginning. However, mid-treatment she suffered a further increase in pain after the aforementioned accident.

\section{Individual results: visual analysis}

What emerges from a visual analysis (see Figure 1) of the individual patient data is a trend characterized by decreased PTSD symptoms, but few other changes and still a persistent degree of distress after treatment. In summary, two patients ( 3 and 5) have reached a relatively low level of symptom severity, two (1 and 4) have had a marked improvement but still suffer considerably, while two (2 and 6) have had unremitting symptoms. For depression, most patients had apparently experienced a decrease, while two ( 1 and 2) seemingly endure continuous distress. Decreased pain and improved functioning can be seen for two patients (3 and 5), and deterioration for two others (1 and 6). Missing data precludes patients 2 and 4 from the analysis of the NOSF-MISS results.

\section{Adherence Challenges}

All therapists reported adherence challenges, mostly due to the patients' needs for help with current stressors or poor compliance. Adherence was evaluated as moderate for four patients $(2,3,5$ and 6$)$ and as low for two (2 and 6).

\section{Evaluation of the combination treatment}

A visual analysis of the symptom progression during the course of the treatment for these six patients does not reveal any clear trend, and there are substantial differences between the patients. Despite the modest decrease in PTSD symptoms observed in tracking them session to session, half of the patients no longer met the criteria for a PTSD diagnosis at the three month follow-up, and two experienced clinically significant change, defined as a decrease of $>2$ SD in CAPS total score. ${ }^{33}$ Furthermore, this was maintained at the six-month follow-up. Regarding depression, only one patient (patient 3) was no longer diagnosed with depression at followup and only one (patient 6) achieved clinically significant change. With respect to pain experience, two patients ( 3 and 5) had decreased pain intensity, two had no change ( 1 and 4) and one (6) experienced increased pain. Patient 2 achieved a clinically significant decrease in pain intensity, when considering the symptom progression from baseline to the final sessions (see Table 1). In 
addition, no differences regarding startup time of the physiotherapy were observed in this study. The treatment results for pain intensity differed substantially between the patients, with some having dramatic changes and others none. Even though three out of five had clinically significant pain reduction at the first follow-up, only two of them retained these reductions three months later. In summary, as a preliminary evaluation, this novel combination treatment appears to be more effective for PTSD symptoms, and less so for pain and depression. However, this argument is highly speculative, for obvious methodological reasons.

\section{Discussion}

Variation in treatment response and potential predictors

It was expected that the combination of NET and physiotherapy would potentially be a promising treatment development for torture survivors with chronic pain and PTSD.

However, as this study failed to illustrate an unambiguous treatment effect on PTSD and pain symptoms, it is clearly not generalizable across different clinical cases. With this research design or actual outcomes, it cannot be concluded that the patients improved because of the treatment provided. Still, it is useful to discuss possible explanations for the differential outcome of our patients, since identifying what factors may predict the outcome is of great importance for clinicians. According to Barlow et al., in case studies, "the issue of interpreting mixed results and looking for causes of failure illustrates an important principle in replication series" (p318). ${ }^{19}$ Failures do not necessarily indicate that a treatment fails to work, but might point to circumstances and conditions under which the treatment does not have the desired effect. ${ }^{19}$ In our study, there are substantial differences in the extent of symptomatic improvement among the patients. In sum, two ("responders": patients 3 and 5) of the patients became much better, with great reductions in both PTSD symptoms and pain intensity. Another two ("part responders": patients 1 and 4) showed improvement with regard to PTSD but still experienced substantial distress, and no change in pain intensity. The last two ("non-responders": patients 2 and 6) did not improve. Why was the treatment more effective for the responders than for the other patients?

Applying Weathers categories, ${ }^{25}$ the responders had lower degrees of PTSD severity prior to treatment (severe and moderate), while the other four had extreme PTSD. One possible explanation is that the treatment is more effective for less severe cases. The results might also reflect a ceiling effect, such that the treatment only has an effect up to a certain degree. Furthermore, the results may reflect a therapist effect, as the same therapist had both patients within each category. The moderate to low degree of compliance or adherence could possibly have led to reduced effect of the treatment for some of the patients. In this study, compliance was not related to symptom load, as the non-responders and responders had the same degree of compliance. Avoidance during exposure was reported in all six cases. However, compliance and adherence were evaluated as lower for the part responders compared with the other patients. In addition, the physiotherapist reported compliance challenges with the part responders, including lack of motivation. Another observation was that the therapists reported extratherapeutic stressors and low physical functioning to interfere with compliance. Thus, better identification of obstacles to compliance might lead to improved outcome for some of the patients. As noted by Silove, ${ }^{26}$ substantial psychosocial problems 
have been associated with poorer treatment outcomes and compliance. Some opponents of trauma-focused therapy for torture survivors argue that it is too narrow and risks ignoring the many and complex issues this group struggles with. ${ }^{27}$ In this regard, Patel ${ }^{28}$ raises concern that this approach is medicalizing a sociopolitical problem, and the cross-cultural applicability of PTSD has been debated. ${ }^{29}$ Thus, we acknowledge that the compliance challenges could be related to cultural or other factors that NET does not adequately address. However, avoidance is one of the core symptoms of PTSD, and a recent paper questions the validity of not offering trauma-focused therapy to refugees based on the assumption that they suffer from complex PTSD due to enduring complex trauma. ${ }^{30}$

The non-responders differed from the others in that both were diagnosed with comorbid social phobia and lifetime panic disorder. Patient 6 additionally suffered from fibromyalgia and recurrent depressive disorder. It is possible that one or both of these disorders negatively influenced treatment outcome, in a similar way to how generalized anxiety disorder affects treatment of other anxiety disorders. ${ }^{31}$ Both disorders can possibly lead to social avoidance or isolation, and thus hinder social support, which is indeed a factor associated with poorer mental health in refugees. ${ }^{32}$

According to Silove, ${ }^{26}$ current stressors might impede recovery. In fact, patient 6 (non-responder) showed improvement in PTSD early on which subsequently declined and instead was aggravated. The aggravation occurred when she received news of her mother's serious disease in her home country and worried about not being able to visit her. At about the same time she had a work-related accident which led to increased pain and reduced mobility and functioning. In addition, all patients except for patient 5 (responder) reported financial struggles related to their health problems. Three patients had fewer financial struggles at the final follow-up.

\section{Limitations of the Study}

First, using an A-B case series design and its accompanied small sample size impedes generalization to other populations. Second, the lack of a control group, only two baseline measurements and no control of eventual parallel pharmacotherapy make it difficult to assess whether the effects found are the consequence of the treatment provided and not owing to common factors, regression to the mean or spontaneous remission. Due to these and a number of other limitations our results should be interpreted with great caution.

\section{Summary and conclusions}

In this study, we have evaluated a novel treatment approach coupling narrative exposure therapy with physiotherapy for torture survivors suffering from chronic pain and PTSD. The small sample size and design precluded generalization and inferences about effect, however enabled an exploratory description of six therapies. Despite its limitations, the study is original in its aim to couple physiotherapy with NET, and demonstrates that some torture survivors who suffer high symptom loads can achieve good outcomes when working in parallel with traumas and pain. Furthermore, we have discussed the possible effect of this combination treatment, with an emphasis on individual differences between the six different cases. It highlights some of the complexities regarding assessment and treatment of torture survivors, exemplifies the point that no treatment works for all patients, and provides tentative explanations 
as for why that might be. Our hope is that it stimulates research that will enable further refinement of rehabilitation strategies for torture survivors.
References

1. Carinci AJ, Mehta P, Christo PJ. Chronic Pain in Torture Victims. Curr Pain Headache Rep. 2010;14(2):73-9.

2. Amris K, Prip K. Physiotherapy for Torture Victims (I): Chronic pain in torture victims: possible mechanisms for the pain. Torture. 2000;10(3):736.

3. Carlsson JM, Mortensen EL, Kastrup M. Predictors of mental health and quality of life in male tortured refugees. Nordic Journal of Psychiatry. 2006;60(1):51-7.

4. Liedl A, Knaevelsrud C. Chronic pain and PTSD: the perpetual avoidance model and its treatment implications. Torture. 2008;18:69-76.

5. Koren D, Norman D, Cohen A, Berman J, Klein EM. Increased PTSD Risk With CombatRelated Injury: A Matched Comparison Study of Injured and Uninjured Soldiers Experiencing the Same Combat Events. Am J Psychiatry. 2005 Feb;162(2):276-82

6. Defrin R, Ginzburg K, Mikulincer M, Solomon $Z$. The long-term impact of tissue injury on pain processing and modulation: A study on ex-prisoners of war who underwent torture. Eur J Pain. 2014 Apr;18(4):548-58.

7. Picavet H, Vlaeyen J, Schouten J. Pain catastrophizing and kinesiophobia: predictors of chronic low back pain. Am J Epidemiol. 2002 Dec 1;156(11):1028-34.

8. Stam R. PTSD and stress sensitisation: A tale of brain and body Part 1: Human studies. Neurosci Biobehav Rev. 2007;31(4):530-57.

9. Geisser ME, Roth RS, Bachman JE, Eckert TA. The relationship between symptoms of posttraumatic stress disorder and pain, affective disturbance and disability among patients with accident and non-accident related pain. Pain. 1996;66(2-3):207-14.

10. Sharp TJ, Harvey A. Chronic pain and post-traumatic stress disorder: mutual maintenance? Clinical Psychology Review. 2001;21(6): 857-877.

11. Asmundson GJG. The emotional and physical pains of trauma: Contemporary and innovative approaches for treating co-occurring PTSD and chronic pain. Depress Anxiety. 2014 Sep;31(9):717-20.

12. Roth RS, Geisser ME, Bates R. The relation of post-traumatic stress symptoms to depression and pain in patients with accident-related chronic pain. J Pain. 2008 Jul;9(7):588-96.

13. NICE. Post-traumatic stress disorder: The management of PTSD in adults and children in primary and secondary care. London: Gaskel and the British Psychological Society; 2005. 
14. Patel N, Kellezi B, de C Williams AC. Psychological, social and welfare interventions for psychological health and well-being of torture survivors. Cochrane Database Systematic Reviews [Internet]. 2014. Available from: http://onlinelibrary.wiley. com/doi/10.1002/14651858.CD009317.pub2/full

15. Weiss W, Ugueto A, Mahmooth Z, Murray L, Hall B, Nadison M, Rasmussen A, Lee JS, Vazzano A, Bass J, Bolton P. Mental health interventions and priorities for research for adult survivors of torture and systematic violence: a review of the literature. Torture 2016;26(1):17-44.

16. Holten N, Prip K, Tived L. Physiotherapy for Torture Survivors: a basic introduction. Copenhagen: International Rehabilitation Council for Torture Victims; 1995.

17. Carlsson JM, Mortensen EL, Kastrup M. A Follow-Up Study of Mental Health and HealthRelated Quality of Life in Tortured Refugees in Multidisciplinary Treatment. J Nerv Ment Dis. 2005 Oct;193(10):651-7.

18. Carlsson JM, Olsen DR, Kastrup M, Mortensen EL. Late mental health changes in tortured refugees in multidisciplinary treatment. J Nerv Ment Dis. 2010 Nov;198(11):824-8.

19. Barlow DH, Nock MK, Hersen M. Single case experimental designs: Strategies for studying behavior change. 3rd ed. Boston: Pearson Allyn \& Bacon; 2009.

20. Office of the United Nations High Commissioner for Human Rights. Istanbul Protocol: Manual on the Effective Investigation and Documentation of Torture and Other Cruel, Inhuman or Degrading Treatment or Punishment. New York and Geneva: United Nations; 2004.

21. Schauer M, Neuner F, Elbert T. Narrative Exposure Therapy: A short Term Treatment For Traumatic Stress Disorders 2nd ed. Cambridge: Hogrefe Publishing; 2011.

22. Kazdin AE. Single-case research designs: Methods for clinical and applied settings. New York: Oxford University Press; 1982.

23. Parsonson BS, Baer DM. The analysis and presentation of graphic data. In: Kratochwill TR, editor. Single-subject research: Strategies for evaluating change. New York: Academic Press; 1978. p101-65.

24. Brossart DF, Parker RI, Olson EA, Mahadevan L. The Relationship Between Visual Analyses in a Simple AB Single-Case Research Design. Behavior Modification. 2006;30(531-563).

25. Weathers FW, Keane TM, Davidson JR. Clinician-administered PTSD scale: a review of the first ten years of research. Depress Anxiety. 2001;13(3):132-56.
26. Silove D. Mental health of asylum seekers: Australia in a global context. In: Allotey P, editor. The Health of Refugees: Public Health Perspectives from Crisis to Settlement. Melbourne, Australia: Oxford University Press; 2003. p. 68-82.

27. Nickerson A, Bryant RA, Silove D, Steel Z. A critical review of psychological treatments of posttraumatic stress disorder in refugees. Clinical Psychology Review. 2011;31(3):399-417.

28. Patel N. Clinical psychology: reinforcing inequalities or facilitating empowerment? The International Journal of Human Rights. 2003;7(1):16-39.

29. Summerfield D. The invention of post-traumatic stress disorder and the social usefulness of a psychiatric category. British Medical Journal. 2001;322:95-8.

30. Heide JJt, Mooren TM, Kleber RJ. Complex PTSD and phased treatment in refugees: A debated piece. European Journal og Psychotraumatology. 2016;7.

31. Wells A. Cognitive therapy of anxiety disorders: A practice manual and conceptual guide. Hoboken, New Jersey, US: John Wiley \& Sons Inc; 1997. p. xiv, 314

32. Lie B. A 3-year follow-up study of psychosocial functioning and general symptoms in settled refugees. Acta Psychiatr Scand. 2002 Dec;106(6):415-25.

33. Jacobson N, Truax P. Clinical significance: a statistical approach to defining meaningful change in psychotherapy research. J Consult Clin Psychol. 1991 Feb;59(1):12-9. 